\title{
Vehicle-bridge interaction system
}

\author{
Daria Provornaya ${ }^{1, *}$, and Sergey Glushkov ${ }^{1}$ \\ ${ }^{1}$ Siberian Transport University, Dusi Kovalchuk st., 191, 630049, Novosibirsk, Russia
}

\begin{abstract}
One of the problems in high-speed railways is the influence and vibrations of bridges caused by moving trains. This problem significantly differs from the problems of road bridges for the following reasons. Firstly, the loads caused by the moving train on the bridge are repeated in nature, as a consecutively rotating loads (wheel), implying that certain frequencies will be caused on the bridge during the passage of the train. In contrast, the loads implied on the road are random in nature, expressed through wheel loads and the distance between the wheels. Secondly, high-speed trains can move at a speed much higher than vehicles moving along the road, which makes it possible for the excitation frequency to coincide with the oscillation frequencies of the bridge, which leads to the so-called resonance effect. Whenever the resonance condition is reached, the reaction of the bridge will be constantly amplified, since there are heavy moving loads passing through the bridge. This effect could hardly be observed on road bridges. Thirdly, the mass ratio of vehicles on the bridge is usually larger due to the fact that the train consists of several coupled cars and the railway track is relatively narrow, it has no more than two tracks in most cases. In contrast, a road bridge may be so large that it can afford four or more lanes for vehicles moving in the opposite direction. For this reason, the interaction between moving vehicles causes a greater reaction for the road bridge than for the railways.
\end{abstract}

\section{Introduction}

The dynamic interaction between a bridge and a moving vehicle is a special discipline in a wide field of structural dynamics. The following vehicles are considered: those that make up the traffic flow of a road bridge, in general, or those that form a connected line of railway cars, in particular. From a theoretical point of view, two subsystems, i.e. a bridge and moving cars, can be modeled as two elastic structures, each of which is characterized by some vibration frequencies. These two subsystems interact with each other with the help of contact forces, i.e. forces caused at contact points between the wheels and the rail surface (railway bridge) or road surface (road bridge). Such problem as this one is non-linear and time-dependent because the contact forces can vary with time, while their values remain constant as a result of the relative motion of the two subsystems. The way in which the two subsystems interact with each other is primarily determined by the inherent frequencies of the two subsystems and the excitation frequency of moving vehicles. The term vehiclebridge interaction (VBI) is used to refer to the interaction between two subsystems.

\footnotetext{
*Corresponding author: molokovada@gmail.com
} 
"Vehicle" is considered as a car, a truck, a tractor trailer, or a railway car that forms part of a train. The term "bridge" is also common. It can be just a beam, continuous construction or a bridge of any type used for roads and railways with or without the effects of surface coverage (for roads) or rails and ballast (for railways). Consideration of VBI is necessary if the vehicle response is taken into account in addition to the bridge response. When designing high-speed railway bridges, for example, the maximum vertical and / or lateral acceleration of moving vehicles is used as indicators to assess the comfort ride of passengers carried by the train.

In addition, the vertical and lateral contact forces of the railway car wheels represent a kind of information for assessing the risk of derailment of trains, especially in the presence of earthquakes. In many cases, in order to overcome a small mass ratio, the elastic and inertial effects of vehicles can be ignored and much simpler models for vehicles can be adopted. One typical example is the modeling of a moving vehicle over the bridge as one moving load, which is called the moving load model and shown in Figure 1. Since the interaction between the two subsystems is not taken into account, the moving load model is only good for computing the response of the larger subsystem (bridge), but not the smaller subsystem (car). The task of a moving load can be considered as a special case in a more general formulation that considers the various dynamic properties of moving vehicles. The goal is to create several effective methods as a part of the finite element method to solve the dynamic response of VBI systems. In the derivation of the basic theories on the basis of analytical approaches or in conducting parametric studies to illustrate the various dynamic effects, more attention will be paid to the problems occurred in the design of high-speed railway bridges in order to reflect concerns about safety and comfort when driving highspeed trains.

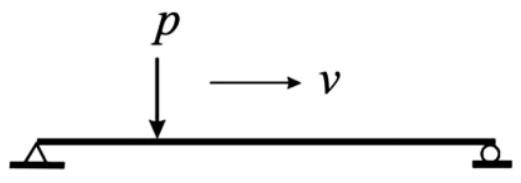

Fig. 1. Moving load model.

\section{Methods of research}

\subsection{Vehicle model}

Neglecting the inertial effect of the vehicle and considering the car as a moving load or pulsating force, Tymoshenko (1922) produced a large number of approximate solutions to the problem of simple beams under moving loads. Similar models were adopted by Eyre and others (1950) and Eyre and Jacobsen (1950) in the study of the dynamic characteristics of two beams, and then by Vellosi (1967) in the study of vibration of suspension bridges. The replacement load model was also adopted by Chen (1978) in analyzing the dynamic characteristics of continuous beams. There are lots of studies of vibration of bridges from moving loads. It is only possible to cite some of the most closely related works, for example, Tan and shore (1968), Friba (1972), Fertis (1973), Sridharan and Mallik (1979), Wu and Dai (1987), Weaver et al. (1990), Galdos et al. (1993), Wang (1997), Zheng et al. (1998), Rao (2000), Chen and Li (2000), and Dugush and Eisenberg (2002). The replacement load model is a simple model, which was often accepted by researchers in studying vibrations of bridges caused by cars. With this model, the main dynamic characteristics of the bridge caused by the action of a moving vehicle can be determined with sufficient accuracy. Nevertheless, the interaction effect between the bridge and the 
moving vehicle was not taken into account. For this reason, the moving load model is only good when the vehicle mass is small in compare with the mass of the bridge, and only when the vehicle response is not taken into account. In cases where the inertia of the vehicle cannot be neglected, the moving mass model shown in Figure 2 should be adopted.

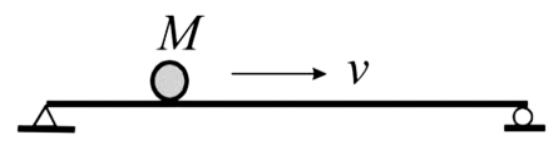

Fig. 2. Moving mass model.

The inertial effects of a beam and a moving vehicle were studied back in 1929 by Jeffcot (1929) using method of successive approximations. Studies in this direction were later carried out by a number of researchers. Hardin (1969) defined the reaction of a simple beam under an arbitrary number of moving masses using the Fourier series expansion. Using the Green function, algorithms for working with moving masses were studied by Ting et al. (1974) and Sadik and Leifolz (1987). For a simple beam with a single moving mass, the solution was obtained by Stanisic (1985) through expanding the eigenfunctions in the series. The moving mass model was developed by Akin and Mofid (1989) in their study of the dynamic response of a beam with various finite conditions using an analytical and numerical approach. One of its disadvantages is that it excludes consideration of the detachment of the active mass on the bridge. This effect will be significant in the presence of disturbances for railway or road, or for vehicles moving at sufficiently high speeds. Sometimes it is necessary to consider the separation and reconnection of a moving vehicle with a bridge for very poor road conditions in which this action of vehicles is very important. The model of the car can still be taken for the Model. In this case, the moving mass is supported by a spring shock absorber, the so-called sprung mass model shown in Figure 3.

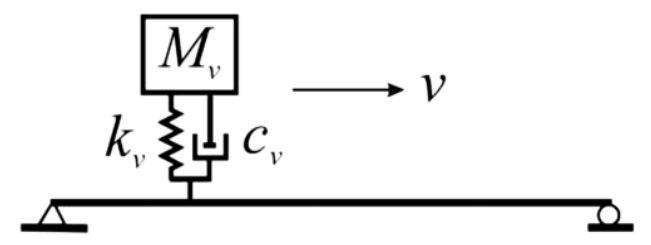

Fig. 3. Sprung mass model.

Biggs (1964) presented a semi-analytic solution of the problem for simple beams and a passing sprung mass. Using the series expansion technique, Pesterev et al. (2001) investigated the response of an elastic joint to several oscillations. Later, Pesterev et al. (2003) studied the asymptotics of the solution of the moving oscillator problem and found that in the limiting case the problem of moving the generator and the moving mass for a simple beam is equivalent in terms of beam movements, but not in terms of beam stresses. Besides, it was shown that for small values of the spring stiffness, the problem of moving the generator is equivalent to the task of a moving load.

With the help of high-performance computers and computing technologies, it becomes possible to have a more realistic modeling of the dynamic properties of the various components that form a moving vehicle. Previously, the effect of an elastic tire and suspension mechanisms was modeled with the help of springs, damping of tire effect, suspension system, and air cushion (Genin et al., 1975, Blejwas et al., 1979; Genin and Chang, 1979; Humar and Kashif, 1993; Green and Kebon, 1994), and energy is dissipated, the effect of mechanism on friction devices (Chatterjee et al., 1994; Tan et al., 1998). Using such methods, a multi-axis trolley or a road-train can be represented as a number of discrete 
masses each supported by several springs and a damper or a friction device. In a study by Yang and co-authors (1999), the railway car was modeled in the form of a rigid beam supported by two sets of spring-damper blocks, each supported by wheels. This model allows us to consider the effect of rocking the car's body. To represent the various dynamic properties of railway freight cars, models of vehicles that contain dozens of degrees of freedom (degrees of freedom) were developed and used by Chu et al. (1986), Wang et al. (1991), Xia and co-authors (2000), and Zhang et al. (2001). To study the "train-rail-bridge" interaction, a train consisting of a sequence of identical cars was considered by others (2001), which assumes that each car should consist of the car's body and rigidly rest on the front and rear trucks. In total, 5 degrees of freedom were obtained on the body of the car, as well as on each truck to account for vertical and lateral movements. In contrast, only three degrees of freedom refer to each wheel pair, which refer to vertical, lateral and rolling movements. Although using a more complex vehicle model can make modeling more realistic, it creates certain computational problems. For example, when modeling bridges, a series of railway cars or vehicles on the road that look like a random stream (Young et al., 1996) are suggested, a discrepancy or convergence can slowly occur during the iteration of a large number of contact forces on wheels / or wheel guides / beams of contact points in a step-by-step analysis. Another problem is that with the help of simplified models it is possible to determine the key parameters that determine the dynamic response of the bridge, which is important for developing rational formulas for use in design (Humar and Kashif, 1993).

\subsection{Model of a bridge}

The beam supported at two ends is the most common construction that has ever been taken to study the vibrations caused by a car. For research projects that rely only on analytical approaches, in principle, there are no restrictions on the type of structures considered for the tasks of VBI, structures can always be represented by finite elements of various forms; the only difference is that a simpler bridge model requires less preparations and efforts in the calculation. In the past, various types of bridges have also been considered in studies of oscillations from vehicles.

The dynamic response of cable-stayed bridges for moving vehicles has been studied by a number of researchers. When modeling a cable-stayed bridge on an elastic base, an approach was proposed to simulate the dynamic effects of the cables. It was suggested to track the levitation of vehicles moving at high speeds. The influence of the roughness of the road surface was examined by Wang and Huang (1992) in studying the vibrations of the cable-stayed bridge. Using an approximate bridge model, taking into account the nonlinear effect of the cables, Jan and Fonder (1998) analyzed the dynamic response of cable-stayed bridges under moving loads. In a review paper by Diane et al. (2000) for the railway run of large-span cable-stayed bridges, he noted that the load has a greater influence in cablestayed bridges than in suspension bridges. Recently, Au and others investigated the effect of the railway load on cable-stayed bridges using various vehicle models, and concluded that the sliding model of mass forces, displacement, and road surface irregularities have a large influence on the bridge response in compare to beam bridges. Guo and $\mathrm{Xu}$ (2001) studied the interaction of a cable-stayed bridge and a tractor trailer moving across the bridge. It was a fully computerized approach. Recently, a mass of a damper system consisting of several subsystems, has been proposed by Yau and Yang (2004) to suppress several resonant peaks of cable-stayed bridges, which can be caused by high-speed trains. Vibration of suspension bridges from road transport was investigated by Chatterjee et al. (1994); vibration and torsion were taken into account. The dynamic interaction between the long suspension bridge, which has a main span length of $1377 \mathrm{~m}$, and the train was 
insignificant - Xia et al. (2000). Later, the suspension bridge was studied by Xu et al. (2003). There are strong winds that act not only on the bridge, but also directly on the train. Their results showed that the bridge's oscillations from the wind adversely affect the safety of a passing train, and also on the comfort of passengers' trip. Another problem in modeling the reaction of the bridge was the consideration of the roughness or irregularities of the railway rails. It has been proven that road surface or irregularities of the road surface can significantly affect the bridge response (Paultre et al., 1992). However, the irregularities of the surface profile mainly depend on the quality of paving, although this problem may also have an accidental character. In most cases, the surface roughness or irregularity of the rail, which is three-dimensional in nature, is often approximated by a two-dimensional profile. As for railways, the profiles of irregularities on the two longitudinal guide rails may be different. The roughness of the road surface was considered by Gupta (1980) for the sine function representing the height of the road surface. To take into account its random nature, the road profile can be modeled as a standard Gaussian random process, and it is generated using a certain power of the spectral density function. Methods like these were widely used by researchers in studying bridge oscillations from cars. The spectral power density functions developed by Dodds and Robson (1973) were modified and used by Wang and Huang (1992) and Huang and others (1993) in analyzes. The work of Marcondis et al. (1991) is of interest because the spectral power density functions are used to compute using data collected from field measurements. This approach was adopted by Jan Lin (1995) in the study of simple and continuous beams, passing vehicles that move at different speeds. As for railway bridges, the irregularities of the rails can be caused by initial errors during installation, settlement of materials. Four geometric parameters can be used to quantitatively describe the irregularities of the rail, i.e. vertical profile, transverse level and alignment (Wiriyachai et al., 1982; Chu et al., 1986; Van et al., 1991). From the point of view of structural dynamics, wavelengths or frequencies from the implied railway disturbances are crucial for the dynamic behavior of the VBI system. The frequencies implied by the roughness of the bridge surface play the same role as the frequency of the bridge, since resonance can occur on the bridge because of passing vehicles.

\section{Solutions}

When studying the dynamic response of the VBI system, two sets of equations of motion can be recorded, one for the bridge and the other for vehicles. It is the interaction or contact forces, which exist when contacting the point of two subsystems, which make two sets of equations in the system. As transition contact points, the matrix system, as a rule, depends on the time that needs to be updated, and is expanded at each time step. Several approaches have been used to solve these two sets of equations. One way is to assume that some offset values for contact points can be solved from the equations of the vehicle. After that, substituting contact forces in the bridge equation, the offset value for contact points can be found. This is so-called iterative approach. Its advantage is that the responses of both vehicles and the bridge at any time can be simultaneously found. Nevertheless, the convergence rate of iterations is going to be low when it comes to a more realistic case of bridges under a large number of vehicles. This can be in a random traffic flow, in highways, or in a connected line, as well as in railways, or when there are twice as much contact points if it is assumed that the vehicle consists of two sets of wheel units.

In the literature, the Lagrange equation with multipliers and constraints was also applied to the analysis of the VBI systems (Blejwas et al., 1979). As is well known, the use of Lagrange multipliers will increase the total number of unknowns and, therefore, will increase the calculations. Speaking theoretically, the third approach is still possible, namely, by eliminating the interaction of contact forces from the initial sets of two 
equations, it is possible to form a new set of coupled equations for the whole VBI system. However, if the approach is performed at the construction level, the symmetry and other advantageous properties of the dynamic matrices, associated with each subsystem, will be destroyed (Jan and Lin, 1995).

Perhaps one of the most effective approaches to solving VBI equations will be the implementation of condensation technique at the element level. Gark and Dukkipathi (1984) used (1965) the reduction of the Guyan scheme for condensation of the degrees of freedom of vehicles. Recently, Jan and Lin (1995) have used dynamic condensation as a way to eliminate all degrees of freedom associated with each vehicle at the element level. Such approaches are good, if only the bridge response (large subsystem) is taken into account. They cannot give exact solutions for the response of mobile vehicles (a smaller subsystem) due to the approximations made in respect of the DOFs car to the DOFs bridge. Other methods that were used to solve the minor differential equations of motion of the VBI problem include: (1) direct integration methods such as Newmark's $\beta$ method (1959), Wilson's $\theta$ method (Sridharan and Mallick, 1979), and Runge -Kutta fourth-order method (Chu et al, 1986); (2) modal superposition method, along with various integration schemes; and (3) the Fourier transform method.

One significant feature of the VBI problem is that the number of vehicles on the bridge depends on the time. The greater the number of vehicles simultaneously operating on the bridge, the higher the level for vehicles to interact with the bridge. To overcome the dependence of the matrix system on the wheel load positions, i.e. place of contact point, one possible approach is to eliminate DOFs in these vehicles and is not in direct contact with the bridge element level of the dynamic condensation method (Yang and Lin, 1995). This will lead to the VBI element that takes into account all the coupling effects. Brief description of the solution presented by Yang and Yau (1997) to obtain the VBI element is presented below. Consider a beam as a certain number of elements under a railway load in which each car is idealized as two concentrated masses, each of which is supported by a spring-loaded damper unit, as shown in Figure 4.

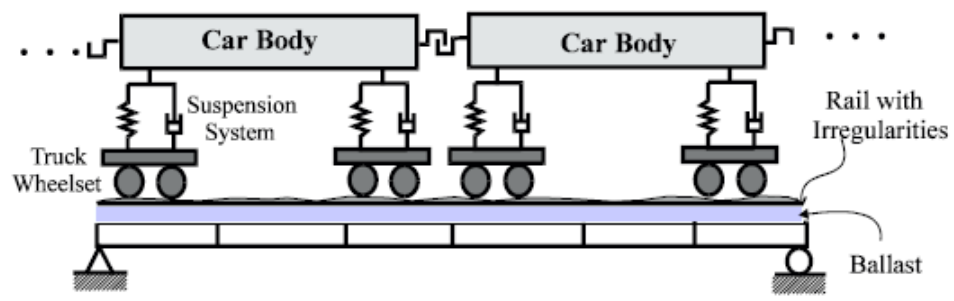

(a)

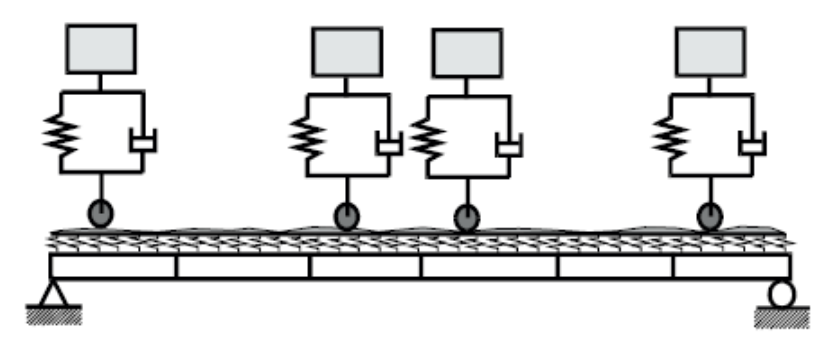

(b)

Fig. 4. Vehicle - bridge system: (a) general model; (b) damper model. 
For the purposes of this study, the interaction element is defined in such a way that it consists of a beam element, the mass of a car, and the mass of a wheel connected by a spring-damper block of a direct action block above the beam element (Figure 5).

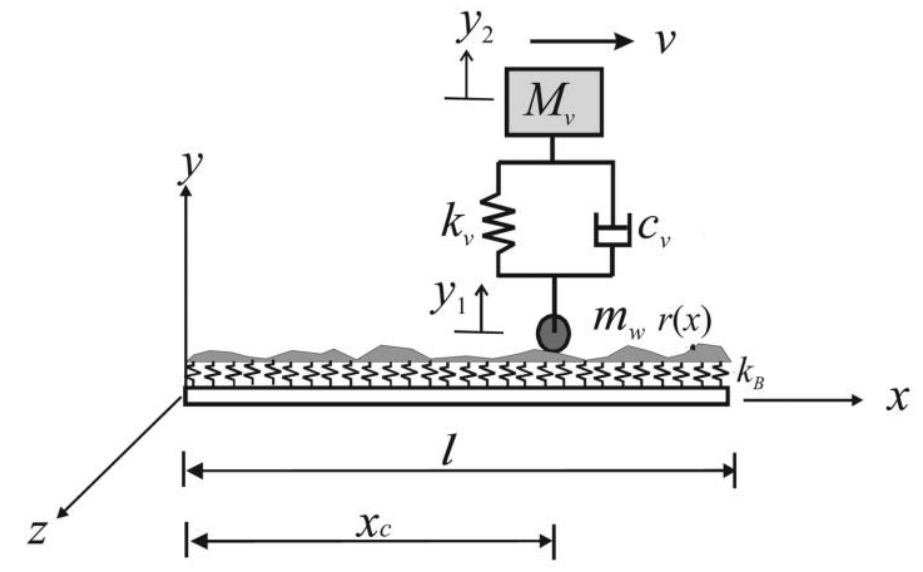

Fig. 5. Vehicle - bridge interaction element.

Parts of the beam that are not directly under the action of the cars can be modeled as ordinary beam elements. Nevertheless, for the remaining parts in direct contact with wheel loads, the interaction elements must be used. With reference to the interaction element shown in Figure 5, two sets of equations of motion can be written, where one is for the beam element and the other for the sprung mass unit.

According to Newmark's single step finite difference formulas, the equation of the sprung mass can be discretized in the time domain from which the DOFs of the vehicle can be solved. Later, by the method of dynamic condensation, DOFs of the sprung mass can condense in the degrees of freedom of the beam element in contact.

This will lead to the VBI element with the interaction effect. Since this element has the same number of degrees of freedom, as the main element, while retaining the symmetry properties in the matrices of the elements, directly assembled with traditional beam elements to form structural equations.

This element is particularly well suited to modeling bridges under a series of mobile sprung mass with a constant or different frequency. There is also the advantage that the response of sprung mass can be obtained at any time of the analysis, which serves as a measure of the comfort of passengers' trip. Using the VBI element, various dynamic properties of beams and vehicles can be considered in development, including irregularities of rails, ballast stiffness, damping of oscillations, and stiffness and damping of suspension devices.

Recently, Jan and Wu (2001) proposed a more universal approach to the solution of the problems of VBI. This method depends on the calculation of the contact forces from the equations of vehicles under conditions of contact displacements. Before this, the vehicle equations must be discretized in the time domain, using difference equations of the Newmark type. Contact forces solved from vehicles can be studied as external loads and presented as node loads to the bridge.

If the equations of the bridge are discretized in time domain, the displacements of the bridge can be solved. This approach was very simple for processing vehicles of varying degrees of complexity that appear in a sequence in which contact forces are involved both vertically and horizontally. 


\section{Results}

In design practice, the dynamic response of the bridge was considered indirectly by increasing the force and stress caused by the static time loads on the impact coefficient, which is defined as the ratio of the maximum dynamic to the maximum static reaction of the bridge to the same load. One of the typical definitions of the impact factor (Yang et al, 1995):

$$
I=\frac{R_{d}(x)-R_{s}(x)}{R_{s}(x)},
$$

where $R_{d}(x)$ and $R_{s}(x)$ are, respectively, the maximum dynamic and static responses of the bridge calculated at the cross-section $\mathrm{x}$.

The responses that can be considered for a beam include deflection, bending moment and transverse force, the definition given in formula (1) is more rational and convenient to calculate than the dynamic increase factor (DIF) proposed by the AASHTO (Manual, 1980), or dynamic amplification factor (DAF), since both the maximum dynamic and static responses are calculated in the same cross section of the bridge. This advantage will become obvious when working with moving loads that appear as a sequence. For example, trains as a random stream, like those on highways, or in solving resonance response problems, in the sense that the reaction of the bridge in the same section will be strengthened, since there are more cargo passing through the bridge. It's necessary to take care and distinguish the maximum exposure factor from the maximum general response calculated for the beam. Factors can be calculated for beams at certain points because the static responses, i.e. the denominator of equation (1), are very small. For this reason, the impact factor calculated or measured for the bridge should not be considered as the only criterion for the design of bridges.

It is well known that a number of factors can affect the bridge during excitation by a moving mobile load, for example, the dynamic properties of the car, the dynamic properties of the bridge, the speed of the car, and the roughness of the road surface. According to AASHTO, technical characteristics and the impact factor are related to the span length $\mathrm{L}$ of the bridge, when the length of the span $\mathrm{L}$ is expressed in feet, and when $\mathrm{L}$ is expressed in meters.

The formula was created several decades ago, based on limited field measurements that are valid for specific types of vehicles and bridges available in those days. If a person realizes that modern trucks are much heavier than those ones which were half a century ago, then the preceding formulas can be convenient for practical design, but they are not the basis of the theory for at least two reasons. Firstly, the formulas are inconsistent in physical units. The impact factor $I$ is a dimensionless value, while the span length has some physical units. Secondly, the use of the span length as a control parameter is not an indication of the physical attribute of the bridge with regard to vehicle-bridge interactions. This is especially true for continuous beams, for which there is more than one span length, none of which can be directly related to the modal form of vibration. Based on the proved more extensive theoretical analyzes and field measurements, it has been shown that impact factors calculated according to current standards can significantly underestimate bridge response in many cases. By designating the speed of a moving vehicle as $V$ and the length characteristic of the beam as $L$, the driving frequency of the moving vehicle can be expressed as $\pi v / L$. The speed parameter $S$, which is particularly useful for expressing the dynamic response of the VBI system, is defined as the ratio of the frequency of a moving vehicle to the main beam frequency, which is dimensionless, that is: 


$$
\mathrm{S}=\frac{\pi V}{\omega L}
$$

\section{Conclusions}

Models of vehicles change and become more complicated with time, including moving load and moving mass. Factors to be taken into account in the analysis of the response of VBI system include the dynamic properties of the engine and the frequencies of moving vehicles, as well as the dynamic properties and roughness of the bridge surface. Despite the higher complexity of vehicle models, for example, consisting of dozens of degrees of freedom, vehicle models and bridge models can be adopted, since it allows identifying key parameters that determine the dynamics of VBI systems. The impact factor is calculated on the basis of the values with the same cross-section of the beam. This is convenient to apply in cases where a number of moving loads are taken into account. These impact formulas, provided by most modern design standards, do not correspond in physical units and do not have a solid theoretical basis, from which the application should not extend on bridges with vehicles passed by them at high speeds. A more rational approach is to link the impact factor, which is dimensionless, to the speed parameter, which is also dimensionless and is defined as the ratio of the frequency of moving vehicles to the oscillation frequency of the bridge.

The problem of VBI is complex because the contact points of vehicles with the bridge move over time. There are various methods for solving this problem. However, the most effective one is based on the condensation of the non-contact vehicle DOFs to the beam element. The element of VBI is obtained in such a way that it can be applied to the solution of a wide variety of the task of VBI, with the help of which the dynamic response of moving vehicles and the bridge can be obtained.

\section{References}

1. Y.B. Yang, J.D. Yau, Y.S. Wu, Vehicle-Bridge Interaction Dynamics With Applications to High-Speed Railways, ISBN 981-238-847-8 (World Scientific Publishing Co. Pte. Ltd)

2. N.A. Donets, Identification of damages in span beam structures of bridges based on the response of vehicles passing through them. Thesis for the degree of Candidate of Technical Sciences (2013)

3. S.P. Glushkov, D.A. Molokova, Technical sciences: theory and practice: collection of materials of the international scientific e-symposium, Monitoring system of damages of bridge structures by integral characteristics, ISBN 978-5-00090-074 (MCNIP, Kirov, 2015)

4. D.A. Promvnaya, S.P. Glushkov, The youth. The science. Technologies: collection of scientific papers of the International Scientific and Technical Conference of Students and Young Scientists in 4 parts, ISBN 978-5-7782-3196-2 (Publishing house of NSTU, Novosibirsk, 2017) 